\title{
Stress: fatores situacionais na central de atendimento ao cliente de uma instituição de ensino superior
}

Stress: situational factors in the customer service center of a higher education institution

Estrés: factores de la situación en el servicio al cliente central de una institución de educación superior

Ana Patrícia Fonseca Coelho Galvão; Rafael Mondego Fontenele ${ }^{2}$; Zulimar Márita Ribeiro Rodrigues $^{3}$

\section{Resumo}

O trabalho por muitas vezes pode desencadear situações estressoras no cotidiano do trabalhador. A central de atendimento de uma instituição, um órgão que frequentemente recebe uma grande demanda de tarefas, este e vários outros fatores podem contribuir para o surgimento do stress no trabalho. Desta forma, o estudo objetiva-se identificar os fatores situacionais que influenciam no surgimento do stress na Central de Atendimento ao Cliente de uma instituição de ensino superior em São Luís - MA. Trata-se de pesquisa descritiva com abordagem quantitativa. Para a coleta dos dados elaborou-se um questionário com perguntas fechadas e Escala de Estresse Percebido (PSS 10). Os principais fatores encontrados no presente estudo foram hora-extra induzida $62 \%$, falta de motivação $60 \%$, falta de reconhecimento $42 \%$, ressarcimento de valores $44 \%$, e carência de benefícios $40 \%$. Com relação ao gênero os homens conseguem perceber o estresse em menor escala na sua vida em geral. Sobre os sintomas físicos, $77 \%$ da amostra têm apresentado dores osteomusculares, cansaço, dores de cabeça e insônia após a jornada de trabalho. Os dados obtidos ajudam a refletir sobre a importância da criação de políticas de prevenção do estresse e promoção da saúde dos trabalhadores.

Descritores: Estresse Ocupacional, Fatores situacionais, Trabalho.

\begin{abstract}
The work for can often trigger stress situations in everyday worker. The call center of an institution, a body that often gets a great demand for jobs, this and many other factors can contribute to the emergence of stress at work. Thus, the objective is to identify situational factors study that affect the appearance of stress in the Customer Service Center of a higher education institution in Sao Luis MA. This is a descriptive research with quantitative approach. To collect the data elaborated a questionnaire with closed questions and Perceived Stress Scale (PSS 10). The main factors in this study were induced overtime $62 \%, 60 \%$ lack of motivation, lack of recognition of $42 \%, 44 \%$ compensation values, and lack of benefits $40 \%$. Regarding gender men can sense the stress on a smaller scale in your life in general. About the physical symptoms, $77 \%$ of the sample have shown musculoskeletal pain, fatigue, headaches and insomnia after the workday. The data help to reflect on

\footnotetext{
${ }^{1}$ Mestra em Saúde e Ambiente (Área de Concentração: Ciências da Saúde) pela Universidade Federal do Maranhão. Docente do Curso de Graduação em Enfermagem da Faculdade Pitágoras - Campus São Luís. Av. Daniel de la Touche, 23 - Olho D'agua, São Luís - MA, 65045-250. E-mail: apfcoelho@ gmail.com.

${ }^{2}$ Especialista em Estratégia Saúde da Família pela Faculdade Gianna Beretta. Enfermeiro Assistencial do Hospital São Domingos. Av. Jerônimo de Albuquerque, 540, Bequimão, São Luís, MA. CEP: 65060-645. E-mail: fhaelmondego@gmail.com.

3 Doutora em Geografia (Área de Concentração: Geografia Humana) pela Universidade de São Paulo. Docente Permanente do Mestrado em Saúde e Ambiente da Universidade Federal do Maranhão - Campus São Luís. E-mail:
} maritaribeiro@ufma.br.
\end{abstract}


ISSN 2179-6750

the importance of creating stress prevention policies and promote workers' health.

Key-words: Occupational Stress, Situational factors, Work.

\section{Resumen}

El trabajo de a menudo puede conducir a situaciones de estrés en los trabajadores todos los días. El centro de llamadas de una institución, un cuerpo que a menudo recibe una gran demanda de puestos de trabajo, este y otros factores que pueden contribuir a la aparición de estrés en el trabajo. Por lo tanto, el objetivo es identificar los factores de situación estudio que afecta a la apariencia de la tensión en el Centro de Servicio al Cliente de una institución de educación superior en Sao Luis MA. Se trata de una investigación descriptiva con un enfoque cuantitativo. Para recoger los datos elaboraron un cuestionario con preguntas cerradas y Escala de Estrés Percibido (PSS 10). Los principales factores en este estudio fueron inducidos horas extraordinarias por $62 \%, 60 \%$ falta de motivación, falta de reconocimiento $42 \%$, los valores de compensación $44 \%$, y la falta de beneficios $40 \%$. En cuanto a los hombres de género pueden percibir la tensión en una escala más pequeña en su vida en general. Acerca de los síntomas físicos, el $77 \%$ de la muestra han mostrado dolor musculoesquelético, fatiga, dolores de cabeza e insomnio después de la jornada laboral. La ayuda de datos para reflexionar sobre la importancia de la creación de políticas de prevención del estrés y la promoción de la salud de los trabajadores.

Palabras-claves: Estrés Laboral, Factores de la Situación, Trabajo.

\section{Introdução}

A palavra stress, utilizada popularmente para ser indicar algo de forma negativa, no entanto, trata-se de uma forma consagrada internacionalmente para representar qualquer estímulo que venha alterar a homeostasia do organismo, seja acelerando ou desacelerando os sistemas orgânicos ${ }^{1}$. Os autores citam em seus estudos, que seu conceito, atribuído por Hans Selye ${ }^{2}$, considerou o stress como um conjunto de reações que o organismo sofre sempre que precisa se adaptar às várias situações do cotidiano, e atualmente tem sido muito associada à sensação de desconforto, sendo cada vez maior o número de pessoas que se definem como estressadas ou relacionam a outros indivíduos na mesma situação.

O stress possui uma característica biológica, ou seja, todo indivíduo irá experimentar em um determinado momento a sensação de estar estressado ${ }^{3}$. O tema se classifica de forma complexa, pois uma vez que estudos relacionam o stress com o surgimento de doenças, especialmente no trabalho, e o crescimento do stress ocupacional se relaciona às exigências do mercado de trabalho ${ }^{4}$.

O termo stress como sendo uma resposta não específica do organismo a qualquer alteração do seu equilíbrio ${ }^{5}$. Resultando de uma interação desajustada, real ou percebida, entre as exigências da situação, os recursos e as capacidades de resposta do indivíduo. No entanto, este desequilíbrio pode ocorrer porque objetivamente as exigências da situação excedem os recursos da pessoa, ou porque esta as percepciona com tal ${ }^{6}$. 
ISSN 2179-6750

A palavra trabalho, de origem latina tripalium, era um instrumento utilizado para torturar criminosos que pagavam seus crimes através do trabalho forçado ${ }^{7}$. Nesse contexto, sua associação com a insatisfação no trabalho como parte da punição que era historicamente atribuída ao homem pelos dominantes do poder ${ }^{8}$. E o desequilíbrio na saúde do trabalhador causado pelo estresse acarreta consequências negativas para as organizações, pois afeta a qualidade dos serviços prestados, aumenta o absenteísmo, o auxílio-doença, a reposição de funcionários, transferências, novas contratações e treinamentos. Devido a estes e outros fatores, "tem crescido a perspectiva de se investigar e se investir na qualidade de vida do trabalhador" 9 .

Trabalho e doença são como uma constante, onde trabalho é capacidade do homem produzir no meio em que vive, e uma vez que há interação entre o homem e a natureza, os dois podem sofrer modificações ${ }^{10}$. Entretanto, o sentido espiritual do trabalho, correlacionando a visão do trabalhador com suas atitudes no trabalho e o aparecimento de doença, seja de ordem física, psíquica ou emocional ${ }^{11}$.

A valorização do ser humano, o respeito à sua integridade e aos seus limites, bem como o estabelecimento de confiança e reconhecimento favorecem a cooperação e comprometimento do trabalhador ${ }^{12}$. Dados obtidos pelo Ministério da Saúde ${ }^{13}$, através da vigilância em saúde do trabalhador, destacam que as condições de trabalho em toda sua plenitude influenciaram no surgimento de distúrbios psíquicos, e ressalta-se a importância de avaliar o stress no trabalho.

Vários aspectos do trabalho podem ocasionar sofrimento psíquico, dentre eles: tipo de ocupação, tempo de exercício, tempo na instituição, tipo de cliente, a pressão sofrida pelos superiores, o relacionamento com os colegas de trabalho, o ambiente físico e até mesmo a falta de autonomia do trabalhador em determinadas situações ${ }^{14}$.

Considerando que o indivíduo produz seus próprios mecanismos de defesa contra o stress, e que, sendo estes compensatórios, estes mecanismos individuais impedem o surgimento de patologias mentais; e quando estes mecanismos são inferiores às demandas de stress, o organismo do trabalhador começa a responder de forma negativa ao trabalho, por meio da desmotivação, insatisfação, falta de atenção e comprometimento no trabalho, e destaca ainda que este estado de stress possa ser caracterizado como um fator de propensão a acidentes de trabalho ${ }^{12}$.

O estresse desenvolve através de um esforço de adaptação, onde o indivíduo tenta mudar sua relação com o agente estressor ${ }^{15}$. E distinguir o indivíduo e seu comportamento torna-se necessário, considerando como elemento importante na dinâmica deste fenômeno, existindo em duas questões centrais sobre o stress em qualquer trabalho, que são a dimensão ou característica da pessoa e a fonte potencial dos agentes no ambiente de trabalho ${ }^{16}$. 
ISSN 2179-6750

Nos ambientes laborais, existem muitas reclamações apontadas, principalmente no que se refere ao atendimento aos clientes que muitas vezes demonstram insatisfação pelos vários serviços oferecidos pelos diferentes mercados comerciais. Além disso, aliam-se sentimentos e descontentamento profissional, a falta absoluta de uma atenção psicológica voltada a essa classe de trabalhadores e os agravos à saúde física e mental que, por ventura, possam estar vivenciando estas pessoas. Esta complexidade de sentimentos, comportamentos e atitudes, chamaram-nos atenção para a necessidade de investigar como stress poderia estar influenciando nas atividades destes trabalhadores.

Destacamos que as diferentes abordagens inerentes ao contexto stress e trabalho, têm propiciado vários questionamentos, suscitando o aparecimento das adversidades, quando se tem capacidade e estímulo para isso. Esta abordagem faz com que o trabalho seja de fato um gerador de prazer, pois deixa de ser uma obrigação para ser algo que dá sentido à vida.

\section{Método}

Trata-se de um estudo descritivo, prospectivo de caráter quantitativo. É descritivo na medida em que atende às características deste tipo de pesquisa, têm por objetivo determinar a distribuição de doenças ou condições relacionadas à saúde, segundo o tempo, o lugar e/ou as características dos indivíduos ${ }^{17}$; e a abordagem quantitativa é caracterizada pelo emprego da quantificação desde a coleta a análise dos dados ${ }^{18}$.

A pesquisa se realizou nas Centrais de Atendimento ao Cliente de uma instituição de ensino superior no município de São Luís-MA. Com uma população constituída de 70 trabalhadores, obteve-se amostra final de 52 trabalhadores. Foram incluídos neste estudo, os trabalhadores de ambos os gêneros que estavam no exercício de suas atividades; e excluídos do estudo, os trabalhadores afastados por motivo de doença, gozando de férias trabalhistas, menores aprendizes ou trabalhadores com período inferior a 3 (três) meses de contratação.

Para a coleta de dados, aplicou-se a Escala PSS 10 (Perceived Stress Scale) traduzida para português. A tradução foi realizada com base no protocolo proposto por Guillemin et al. (1993). A PSS 10 possui 10 questões com opções de resposta que variam de zero a quatro $(0=$ nunca; $1=$ quase nunca; $2=$ às vezes; $3=$ quase sempre $4=$ sempre). As questões com conotação positiva $(4,5,7$ e 8$)$ têm sua pontuação somada invertida, da seguinte maneira, $0=4,1=3,2=2$, $3=1$ e $4=0$. As demais questões são negativas e devem ser somadas diretamente. O total da escala é a soma das pontuações destas 10 questões e os escores podem variar de zero a 40 pontos. Aplicou-se ainda, um questionário contendo 15 perguntas fechadas para coleta de dados socioeconômicos e demais 
ISSN 2179-6750

aspectos importantes para alcance dos objetivos do estudo. Realizou-se a pesquisa no mês de maio de 2015 e desenvolvida através das seguintes etapas: a) seleção da amostra e convite para participação da pesquisa; b) explicação dos objetivos e considerações éticas que envolviam o estudo.

Os trabalhadores foram entrevistados em seu local de trabalho, de acordo com suas disponibilidades no dia da pesquisa. Os dados obtidos foram agrupados de acordo com sua especificidade, em função de sua preferência absoluta e dos índices percentuais analisados, compilados e apresentados na forma de tabelas no programa STATA ${ }^{\circledR}$, versão 10.0. O presente estudo obedeceu aos aspectos éticos e legais de pesquisa envolvendo seres humanos, de acordo com as recomendações do Conselho Nacional de Saúde (CNS) e a Resolução 466/12, sendo submetido à Plataforma Brasil e após análise documental, aprovado no Comitê de Ética em Pesquisa no dia 03.03.2015.

\section{Resultados e Discussão}

Os resultados serão apresentados em razão de sua frequência absoluta. Na primeira segunda seção serão discutidos os dados percentuais obtidos pelo questionário de autoria dos pesquisadores. Na segunda seção serão discutidos os dados obtidos através da Escala de Estresse Percebido (PSS $10)$.

Foram entrevistados 52 trabalhadores da Central de Atendimento ao Cliente de uma instituição de ensino superior, em três campi localizados no município de São Luís-MA. Após a análise dos dados, os mesmos foram organizados através de tabelas para melhor visualização e compreensão.

Tabela 1. Distribuição do perfil socioeconômico dos trabalhadores.

\begin{tabular}{|c|c|c|c|}
\hline Variável & Categoria & n & $\%$ \\
\hline \multirow[t]{2}{*}{ Gênero } & Masculino & 12 & 23 \\
\hline & Feminino & 40 & 77 \\
\hline \multirow[t]{3}{*}{ Idade } & $18-23$ & 11 & 21 \\
\hline & $24-29$ & 31 & 60 \\
\hline & 30 ou mais & 10 & 19 \\
\hline \multirow[t]{3}{*}{ Estado Civil } & Casado & 08 & 15 \\
\hline & Solteiro & 34 & 66 \\
\hline & União estável & 10 & 19 \\
\hline \multirow[t]{4}{*}{ Escolaridade } & Nível médio & 04 & 8 \\
\hline & Nível médio técnico & 00 & 0 \\
\hline & Superior incompleto & 24 & 46 \\
\hline & Superior completo & 24 & 46 \\
\hline \multirow[t]{4}{*}{ Renda Mensal } & Até R\$1.000,00 & 26 & 50 \\
\hline & De $R \$ 1.000,00$ a $1.500,00$ & 14 & 27 \\
\hline & De $R \$ 1.500,00$ a $2.000,00$ & 11 & 21 \\
\hline & Acima de $\mathrm{R} \$ 2.000,00$ & 01 & 2 \\
\hline Total & & 52 & 100 \\
\hline
\end{tabular}


ISSN 2179-6750

Na tabela 1, observa-se a distribuição do perfil socioeconômico dos sujeitos da pesquisa, após análise dos dados que 77\% (40) dos trabalhadores são do gênero feminino, $66 \%$ (34) solteiros, com média de idade entre 24 e 29 anos. Com relação à formação acadêmica 46\% (24) dos entrevistados possuem o ensino superior completo, enquanto que outros $46 \%$ (24) possuem o ensino superior incompleto e a renda mensal média dos sujeitos é de até $\mathrm{R} \$ 1.000,00$. Nota-se que $46 \%$ (24) da amostra ainda não concluíram o ensino superior, desta forma, o presente estudo concorda com pesquisa realizada em São Paulo, onde a relação trabalho e estudo é um fator que pode desencadear o processo de stress, uma vez que os trabalhadores com o ensino superior incompleto no presente estudo estão com a graduação em curso e enfrenta a longa jornada diária entre trabalho e universidade, engarrafamentos, alimentações fora dos horários e de forma inadequada ${ }^{19}$.

A renda mensal para 50\% (26) dos entrevistados está pouco acima de 01 salário, o que corrobora com outro estudo desenvolvido, onde a baixa remuneração é apontada pelos trabalhadores como um dos maiores fatores que influenciam o aparecimento de estresse no trabalho 20

Tabela 2. Detecção do nível de estresse com relação ao gênero através do Escore da PSS 10.

\begin{tabular}{|l|l|c|c|}
\hline Variável & Categoria & $\mathbf{n}$ & $\%$ \\
\hline & Masculino Escore $<10$ & 03 & 25 \\
\hline & Masculino Escore 11 - 25 & 08 & 67 \\
\hline & Masculino Escore >26 & 01 & 8 \\
\hline & Total & $\mathbf{1 2}$ & \\
\hline & Feminino Escore $<10$ & 02 & 5 \\
\hline & Feminino Escore $11-25$ & 31 & 77,5 \\
\hline & Feminino Escore $>26$ & 07 & 17,5 \\
\hline Total & Total & $\mathbf{4 0}$ & \\
\hline Fonte: Pesquisa direta & $\mathbf{5 2}$ & $\mathbf{1 0 0}$ \\
\hline
\end{tabular}

A Escala de Estresse Percebido (PSS 10) não é um instrumento para diagnóstico do estresse ocupacional, caracteriza-se por apenas uma medida genérica dos níveis de estresse que o próprio indivíduo consegue perceber acerca de sua vida em geral. Resultados de estudos com a PSS normalmente são representados por níveis de estresse, onde os escores são categorizados em estresse baixo, estresse médio e estresse alto. Para os autores da PSS, essa categorização pode ocasionar perda da precisão estatística ${ }^{21}$.

Optou-se pela divisão em três níveis de escores para melhor entendimento da PSS 10, compreendendo que quanto maior a pontuação do escore nesta escala, maior é a percepção do indivíduo sobre o estresse em sua vida. Os níveis definidos neste estudo foram: menor que 10 pontos, entre 11 e 25 pontos, e maior que 26 pontos. 
ISSN 2179-6750

Pode-se observar na tabela 2, que 17,5\% (7) dos trabalhadores entrevistados do gênero feminino pontuaram escores maiores que 26 pontos na PSS 10, enquanto $8 \%$ (1) dos trabalhadores do gênero masculino pontuaram a mesma medida, onde o gênero feminino também pontuou escores altos na Escala de Estresse Percebido ${ }^{22}$.

Observamos que os maiores escores pontuados pela PSS 10 no presente estudo para os dois gêneros está entre 11 e 25 pontos, onde para os trabalhadores do gênero masculino esta média representa $67 \%$ (8) e para o gênero feminino 77,5\% (31). Estes achados para o gênero feminino podem estar associados pela frequência da sobrecarga de tarefas específicas do mundo feminino, caracterizando a jornada de trabalho com os afazeres domésticos em dupla ou tripla jornada de trabalho ${ }^{23}$.

Tabela 3. Identificação das situações e fatores que influenciam no surgimento do stress.

\begin{tabular}{|c|c|c|c|}
\hline Variável & Categoria & n & $\%$ \\
\hline \multicolumn{4}{|l|}{ Fatores } \\
\hline \multirow[t]{3}{*}{ Hora Extra } & Espontânea & 8 & 15 \\
\hline & Mediada & 12 & 23 \\
\hline & Induzida & 32 & 62 \\
\hline \multirow{3}{*}{ Trabalho em Equipe } & Nem sempre & 30 & 58 \\
\hline & Na maioria das vezes não & 12 & 23 \\
\hline & Sim & 10 & 19 \\
\hline \multirow{3}{*}{ Motivação Profissional } & Nunca & 31 & 60 \\
\hline & Esporadicamente & 16 & 31 \\
\hline & Sempre & 5 & 9 \\
\hline \multirow[t]{3}{*}{ Humor do Cliente } & Nem sempre & 25 & 48 \\
\hline & Na maioria das vezes sim & 19 & 37 \\
\hline & Nunca & 8 & 15 \\
\hline \multirow[t]{7}{*}{ Sentimentos Negativos } & Cliente estressado & 18 & 34 \\
\hline & Suporte a outro setor & 15 & 29 \\
\hline & Troca de turno & 1 & 2 \\
\hline & Nunca experimentei & 3 & 6 \\
\hline & Fim do intervalo & 2 & 4 \\
\hline & Cliente com muitas dúvidas & 1 & 2 \\
\hline & Não posso revidar & 12 & 23 \\
\hline \multicolumn{4}{|l|}{ Situações } \\
\hline \multirow[t]{8}{*}{ Circunstância e ambiente } & Burocracia dos processos & 7 & 13 \\
\hline & Mobília inadequada & 10 & 19 \\
\hline & Escala de trabalho & 2 & 4 \\
\hline & Falhas no sistema & 1 & 2 \\
\hline & Repetitividade de tarefas & 3 & 6 \\
\hline & Não considero fatores estressores & 3 & 6 \\
\hline & Dependência de outros setores & 4 & 8 \\
\hline & Falta de reconhecimento & 22 & 42 \\
\hline \multirow[t]{6}{*}{ Dificuldade em manter a calma } & Ressarcimento de valores & 23 & 44 \\
\hline & Cobrança indevida & 9 & 17 \\
\hline & Retificação de documento & 0 & 0 \\
\hline & Emissão de documentos & 3 & 6 \\
\hline & Entrevista PROUNI e FIES & 16 & 31 \\
\hline & Nunca perco a calma & 1 & 2 \\
\hline \multirow[t]{2}{*}{ Dores, insônia, cansaço } & Sim & 40 & 77 \\
\hline & Não & 12 & 23 \\
\hline Total & & 52 & 100 \\
\hline
\end{tabular}


Na tabela 3 descrevemos as situações e fatores que favorecem o surgimento do estresse no trabalho independente do gênero dos trabalhadores, quando questionados sobre os fatores causadores do stress, a principal causa relatada por $62 \%$ (32) da amostra foi hora-extra induzida, onde o trabalhador relata ser forçado a permanecer na instituição devido à sobrecarga de atividades, necessitando ampliar sua jornada de trabalho para cumprir com suas responsabilidades dentro do prazo.

Observou-se que nem sempre se prioriza o trabalho em equipe, esse dado correspondeu a $58 \%$ (30) da amostra total, e ainda 60\% (31) destes trabalhadores nunca se sentiram motivados pela empresa, no entanto $48 \%$ dos sujeitos não são influenciados pelo humor dos clientes, mas 34\% (18) experimentam sentimentos negativos (ansiedade, raiva, angústia) quando o cliente já chega estressado, embora 29\% (15) tenham relatado experimentar estes sentimentos quando são convocados a dar suporte a outro departamento na instituição.

Quando questionados sobre as situações do dia-a-dia que mais produziam a sensação de desgaste físico e emocional 19\% (10) relatou que a mobília é inadequada e $42 \%$ (22) referiu a falta de reconhecimento pelo seu trabalho.

Os trabalhadores foram questionados ainda sobre os momentos em que mais perdem a calma no trabalho, e as situações mais apresentadas foram as solicitações de ressarcimento de valores $44 \%$ (23) e o período de entrevistas para o ProUni - Programa Universidade Para Todos e FIES Financiamento Estudantil 31\% (16), que por sua vez são Programas do Governo Federal para acesso ao ensino superior, sujeitos à comprovação de baixa renda. Estes trabalhadores quando questionados sobre a presença de sintomas físicos 77\% (40) relataram ter apresentado dores osteomusculares, cansaço, dores de cabeça, má digestão ou insônia ao final do dia.

Em estudos desenvolvidos, relacionam o reconhecimento no trabalho com a autorrealização, desta forma o trabalhador que não é reconhecido consequentemente não se sentirá motivado ${ }^{24}$. Entretanto, um estudo evidenciou impacto que o conflito de relacionamento entre a equipe proporciona no ambiente laboral como fatores situacionais para o estresse, destacado pela maioria dos entrevistados no presente estudo com a falta de trabalho em equipe ${ }^{2}$.

A mobília inadequada referida por $19 \%$ (10) da amostra do presente estudo pode estar associada à quantidade de horas sentadas no trabalho e a longa jornada como elementos fundamentais na interferência da qualidade de vida dos trabalhadores. Este fato pode ser agravado com a falta de conforto relatada pelos trabalhadores relacionada à mobília que muitas vezes apresenta condições inadequadas para o trabalho ${ }^{25}$. 
Tabela 4. Estratégias de enfrentamento ao stress e melhorias das condições de trabalhos sugeridas pelos trabalhadores.

\begin{tabular}{|l|l|c|c|}
\hline Variável & Categoria & n & \% \\
\hline Satisfação no Trabalho & Aumento de salário & 7 & 13 \\
\hline & Benefícios (sala de repouso, ticket, etc.) & 21 & 40 \\
\hline & Diminuição da jornada de trabalho & 5 & 10 \\
\hline \multirow{2}{*}{ Manejo do Stress } & Aumento do quadro de funcionários & 19 & 37 \\
\hline & Chorar & 2 & 4 \\
\hline & Pensar em pedir demissão & 14 & 27 \\
\hline & Ficar de mau humor & 10 & 19 \\
\hline & Tem insônia & 12 & 23 \\
\hline & Sair a lazer & 11 & 21 \\
\hline & Trocar de setor & 0 & 0 \\
\hline & Nunca me percebi estressado & 3 & 6 \\
\hline & & $\mathbf{5 2}$ & $\mathbf{1 0 0}$ \\
\hline
\end{tabular}

Fonte: Pesquisa direta

Percebemos na tabela 4, as estratégias desenvolvidas para melhorar as condições de trabalho propostas pelos trabalhadores, onde 13\% (7) dos sujeitos da pesquisa sugeriram aumento de salário, porém os maiores índices percentuais foram representados por $40 \%$ (21) dos trabalhadores que sugeriram o fornecimento de maiores benefícios como uma sala de repouso, refeitório, ticket alimentação, dentre outros, e por 37\% (17) dos trabalhadores que propuseram o aumento do quadro de funcionários.

Pesquisas associaram a insatisfação no trabalho com a baixa remuneração oferecida ${ }^{26}$, no entanto outros autores ressaltam esta insatisfação com o ambiente físico, dado que o presente estudo corrobora com a falta de suporte oferecido pela instituição no sentido de que o trabalhador não possui local para realizar sua alimentação e repousar no horário de intervalo ${ }^{27}$.

Percebe-se ainda que $27 \%$ (14) dos trabalhadores de ambos os gêneros, já pensaram em pedir demissão de seus cargos, frente às situações estressoras apresentadas 19\% (10) ficam de mau humor até o final da jornada de trabalho o que pode diminuir a qualidade do atendimento oferecido, e após a jornada de trabalho diária 23\% (12) dos trabalhadores têm apresentado diminuição da qualidade do sono. Esta diminuição da qualidade do sono também foi evidenciada em estudos quando correlacionada com o estresse no trabalho ${ }^{28}$.

\section{Conclusão}

Este trabalho permitiu a importância de se compreender o stress e de que forma essa relação ambiente de trabalho-mundo são ligadas, onde não podemos culpar a sociedade, visão coletiva, muito menos o homem de forma individual. Saber conduzir o stress, e o resultado o resultado desta relação é de responsabilidade de todos.

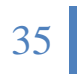


ISSN 2179-6750

Nesta pesquisa podemos concluir que, todos os fatores que mais influenciam no desencadeamento de situações estressoras que lidam diariamente com atendimento ao cliente são a sobrecarga de atividades, evidenciadas por $62 \%$ dos trabalhadores e pela necessidade de fazer horaextra para conseguir atender as demandas da instituição.

Ressaltamos a importância de ajudar os trabalhadores a se conscientizarem sobre seus objetivos no trabalho, entretanto a falta de harmonia entre a equipe, declarada por $58 \%$ dos entrevistados, tornando o ambiente laboral desagradável; a falta de motivação por parte da empresa, relatada por $60 \%$ dos trabalhadores; a falta de reconhecimento pelo trabalho realizado, demonstrada por $42 \%$ são situações geradoras de stress, além de outros problemas relatados como a mobília que impede a postura adequada 19\%, gerando dores osteomusculares, cansaço e insônia $77 \%$.

Diante dos dados obtidos, percebemos a necessidade de refletir sobre a importância da criação de políticas de prevenção do stress e promoção da saúde no trabalho, além de conscientização do corpo administrativo das instituições quanto a medidas preventivas e minimizadoras do stress, influenciando diretamente na qualidade de vida no trabalho bem como na qualidade do serviço oferecido pelas instituições, consequentemente diminuindo da rotatividade devida troca de setor, demissões e ainda afastamento por motivo de doenças destes funcionários.

\section{Referências}

1. Limongi-França AC, Rodrigues AL. Stress e trabalho: uma abordagem psicossomática. 2 ed. São Paulo: Atlas, 1999.

2. Costa DT, Martins MCF. Estresse em profissionais de enfermagem: impacto do conflito no grupo e do poder do médico. Rev Esc de Enferm USP, 45, n. 5, p. 1191-1198, 2011.

3. Ballone GJ, Pereira Neto E, Ortolani IV. Da emoção à lesão: um guia de medicina psicossomática. São Paulo: Manole, 2002.

4. Ramos P. Síndrome de Burnout: estresse ocupacional. Revista Vida e Saúde, p. 12-14, 2005.

5. Vaz-Serra A. O Stress na vida de todos os dias. 2 ed. Coimbra: Edição do autor, 2002.

6. Lazarus R, Folkman S. Stress, appraisal and coping. New York: Springer, 1984.

7. Bom Sucesso E. Trabalho e qualidade de vida. 2 ed. Rio de Janeiro: Qualitymark/ Dunya, 2005.

8. Gallo CMC. Desvelando fatores que afetam a satisfação e insatisfação no trabalho de uma equipe de enfermagem [Dissertação]. Rio Grande: Universidade Potiguar, 2003.

9. Pereira AMB. Burnout: quando o trabalho ameaça o bem-estar do trabalhador. São Paulo: Casa do Psicólogo, 2002. 
ISSN 2179-6750

10. Mello Filho J. Concepção psicossomática: visão atual. São Paulo: Casa do Psicólogo, 2002.

11. Boog G. Manual de gestão de pessoas e equipes. São Paulo: Gente, 2005. vol. I e II.

12. Dejours, JC. A loucura do trabalho: estudo de psicopatologia do trabalho. 5 ed. Cortez Editora. Oboré, 1992.

13. Ministério da Saúde. Caderno de Atenção Básica no 05 - Saúde do trabalhador. Brasília: Ministério da Saúde, 2002.

14. Benevides-Pereira AMT et al. Burnout: quando o trabalho ameaça o bem-estar do trabalhador. 4 ed. São Paulo, Casa do Psicólogo, 2010.

15. Antoniazzi AS, Dell'aglio DD, Bandeira DR. O conceito de coping: uma revisão teórica. Estudos de Psicologia, p. 273-294,1998.

16. Lipp M. Stress e suas implicações. Revista Psicologia. v. 4, n.3, p. 5-19, 2000.

17. Seltiz J, Deutsch C. Métodos de pesquisa nas relações sociais. São Paulo: Edusp, 1975.

18. Lobiondo-Wood G, Haber J. Pesquisa em enfermagem: métodos, avaliação crítica e utilização. 4. ed. Rio de Janeiro: Guanabara Koogan, 2001.

19. Silva VLS, Chiquito NC, Andrade RAPO, Brito MFP, Camelo SHH. Fatores de estresse no último ano do curso de graduação em enfermagem: percepção dos estudantes. Rev. enferm. UERJ, Rio de Janeiro, jan/mar; 19(1):121-6, 2011.

20. Mizobuchi LEC, Cury CFMR. Estresse na enfermagem: mensuração das situações geradoras em um hospital geral. Rev. Ins Ciências Saúde. v. 25, n. 4 , p. 349-355, 2007.

21. Luft CB, Sanches SO, Mazo GZ, Andrade A. Versão brasileira da escala de estresse percebido: tradução e validação para idosos. Rev de Saúde Pública. v. 41, n. 4, p. 606-615, 2007.

22. Medeiros Neto CF, Almeida GA, Ramos BC, Costa SKP, Silva HPA, Sousa MBC. Análise da percepção da fadiga, estresse e ansiedade em trabalhadores de uma indústria de calçados. J Bras Psiquiatr. v. 61, n. 3, p.133-138, 2012.

23. Seleghim MR, Mombelli MA, Oliveira MLF, Waidman MAP, Marcon SS. Sintomas de estresse em trabalhadoras de enfermagem de uma unidade de pronto socorro. Rev Gaúcha Enferm. v. 33, n. 3, p. 165-173, Porto Alegre, 2012.

24. Bendassolli P. Reconhecimento no trabalho: perspectivas e questões contemporâneas. Rev. Psicologia em Estudo, v. 17, n. 1, p. 37-46, jan./mar. Maringá, 2012.

25. Grande AJ, Silva V, Rocha TBX, Vilela Junior GB. Determinantes da qualidade de vida no trabalho: ensaio clínico controlado e randomizado por clusters. Rev Bras Med Esporte. v. 19, n. 5, set/out, 2013. 
ISSN 2179-6750

26. Ruviaro MFS, Bardiagi MP. Síndrome de Burnout e satisfação no trabalho em profissionais da área de enfermagem do interior do RS. Revista do Departamento de Ciências Humanas da UNISC. Barbarói, 33, p. 194-216, 2011.

27. Sá AMS, Martins-Silva PO, Funchal B. Burnout: o impacto da satisfação no trabalho em profissionais de enfermagem. Rev. Psicologia \& Sociedade. v. 26, n. 3, p. 664-674, 2014.

28. Rocha MCP, Martino MMF. O estresse e qualidade de sono do enfermeiro nos diferentes turnos hospitalares. Rev. Escola de Enfermagem da USP. v. 44, n. 2, p. 280-286, São Paulo, 2010. 